\title{
In-plane coherent control of plasmon resonances for plasmonic switching and encoding
}

\author{
Liyong Jiang ${ }^{1,2}$, Tingting Yin², Alexander M. Dubrovkin², Zhaogang Dong $\mathbb{1}^{3}$, Yuntian Chen ${ }^{4}$, Weijin Chen ${ }^{4}$, \\ Joel K. W. Yang $\mathbb{B}^{3,5}$ and Zexiang Shen ${ }^{2}$
}

\begin{abstract}
Absract
Considerable attention has been paid recently to coherent control of plasmon resonances in metadevices for potential applications in all-optical light-with-light signal modulation and image processing. Previous reports based on out-ofplane coherent control of plasmon resonances were established by modulating the position of a metadevice in standing waves. Here we show that destructive and constructive absorption can be realized in metallic nano-antennas through in-plane coherent control of plasmon resonances, which is determined by the distribution rule of electricalfield components of nano-antennas. We provide proof-of-principle demonstrations of plasmonic switching effects in a gold nanodisk monomer and dimer, and propose a plasmonic encoding strategy in a gold nanodisk chain. In-plane coherent control of plasmon resonances may open a new avenue toward promising applications in optical spectral enhancement, imaging, nanolasing, and optical communication in nanocircuits.
\end{abstract}

\section{Introduction}

Over the past few years, significant efforts have been devoted to studying the strong light-matter interactions in plasmonic systems at nanoscale ${ }^{1}$. Based on the control of localized surface plasmon resonance (LSPR), many practical applications have been reported, including surface-enhanced Raman scattering ${ }^{2,3}$, plasmon waveguides $^{4}$, molecular rulers ${ }^{5}$, biosensing and bioimaging ${ }^{6,7}$, surface-enhanced fluorescence ${ }^{8,9}$, nanolasers ${ }^{10-12}$, plasmonic color printers ${ }^{13,14}$, plasmonic tunnel junctions ${ }^{15}$, and plasmonic holography and metalens ${ }^{16-18}$. In these pioneering works, the control of plasmon resonances focused on designing the configurations of plasmonic nanostructures. People already understood the size- and

Correspondence: Liyong Jiang (jly@njust.edu.cn) or

Joel K.W. Yang (joel_yang@sutd.edu.sg) or Zexiang Shen (zexiang@ntu.edu.sg) 'Department of Physics, School of Science, Nanjing University of Science and Technology, Nanjing 210094, China

${ }^{2}$ Centre for Disruptive Photonic Technologies, The Photonics Institute, School of Physical and Mathematical Sciences, Nanyang Technological University, 21 Nanyang Link, Singapore 637371, Singapore

Full list of author information is available at the end of the article. shape-dependent ${ }^{19,20}$ LSPR of single plasmonic nanoparticles and coupled plasmonic systems ${ }^{21,22}$ based on the classical Mie theory ${ }^{23}$ and well-established plasmonic hybridization models ${ }^{24-26}$. The resonant wavelength of the fundamental LSPR is proportional to the single nanoparticle's size, which is below the quasi-static approximation limitation. The bright and dark plasmon resonances, as well as the Fano-like ${ }^{27}$ and electromagnetically induced transparency ${ }^{28}$ phenomena in complex plasmonic systems, are determined by the LSPR coupling and energy transfer.

Moreover, in conventional optical studies of single and coupled nano-antennas, the light beam usually illuminates normally to the sample surface from one direction. As a result, the control of plasmon resonances can also be realized under asymmetric out-of-plane illumination conditions by changing incident light parameters, including polarization, amplitude, and phase, or by using a pulsed-beam and single-photon source for ultrafast ${ }^{29}$, nonlinear $^{30}$, and quantum optical study ${ }^{31}$. Recently, coherent control of plasmon resonances under 
symmetrical out-of-plane illumination has opened a new way of signal modulation. By changing the position of a metadevice in standing waves, interaction between light and the metadevice can reach the maximum and minimum at the antinode and node, respectively. Based on an out-of-plane interferometric setup, coherent perfect absorption $^{32,33}$ and transparency in plasmonic metasurfaces have been demonstrated to show novel applications in optical communication, such as all-optical lightwith-light coherent modulators ${ }^{34}$, optical amplifiers ${ }^{35}$, and arithmetic units ${ }^{36}$. However, the out-of-plane coherent control of plasmon resonances has shown obvious limitations in mode and spatial selection. For example, optical response is generally uniform for nano-antennas on the metadevice due to isotropy-coherent absorption in the sample plane normal to the incidence.

Here we first report a study on in-plane coherent control of plasmon resonances in typical metallic nanoantennas. It has been widely reported that for nanoantennas, the plasmon resonances at oblique incidence $^{37-40}$ are quite different from those under normal incidence due to the retardation effect ${ }^{41}$, which indicates anisotropy optical response in the sample plane under a symmetric in-plane illumination condition. We show selectively multimode coherent absorption in a gold nanodisk monomer and dimer, as well as spatial selection of coherent absorption in a gold nanodisk chain. We provide proof-of-principle demonstrations of plasmonic switching and encoding applications based on the mode and spatial selection of coherent absorption in single and coupled gold nanodisks.

\section{Results}

\section{Setups for in-plane coherent control of plasmon resonances}

Two possible setups are proposed here for in-plane coherent control of plasmon resonances through changing the symmetry of in-plane illumination. One is based on a fiber-waveguide interferometer (Fig. 1a), where completely symmetrical in-plane illumination is constructed by coupling two polarized coherent beams from the single-mode fiber-connected objective lens into the input waveguides without phase delay, whereas asymmetrical in-plane illumination can be realized by blocking one input beam (half-illumination) or introducing a phase delay between two beams. This setup can be widely used to study the in-plane coherent control of plasmon resonances in all kinds of plasmonic nanostructures with axial symmetry. The out-of-plane far-field signal carrying the LSPR information will be collected by an objective lens. However, this kind of setup still faces challenges in experiments, including accurate phase control required between two input fibers and high coupling efficiency required between fibers and waveguides. As a comparison, the widely used dark-field (DF) confocal microscopy is more convenient (Fig. 1b), where the polarized light is focused onto the sample through a condenser lens with an annular aperture and the backward scattering light is collected by an objective lens in a confocal setup. In this setup, completely symmetrical in-plane illumination can be easily satisfied once the input light is focused onto the center of the sample, whereas asymmetrical in-plane illumination (e.g., quarter illumination) can be constructed by blocking three-fourth of the area of the annular aperture. It is clear that such a DF illumination setup still contains out-of-plane wave vector components; thus, a relatively large numerical aperture (NA) of the condenser lens is required to reach the grazing incident condition. This setup is suitable for studying plasmonic nanostructures with sizes comparable to the focused spot size of the incident light beam.

\section{In-plane coherent control of plasmon resonances with destructive and constructive interference}

Based on the fiber-waveguide interferometer, we theoretically studied the in-plane coherent control of plasmon resonances in gold nanodisks. Figure $2 \mathrm{a}, \mathrm{b}$ shows the calculated absorption spectra of gold nanodisk monomers on $\mathrm{SiO}_{2} / \mathrm{Si}$ substrate with diameter ranging from 140 to $200 \mathrm{~nm}$. For each gold nanodisk, the $s$-polarized plan wave was introduced from the right side (dashed line), generating one fundamental LSPR ("F") peak and one retardation-induced high-order plasmon resonance ("H") peak, both of which continuously red-shift with increasing diameters of gold nanodisks ${ }^{41}$. When symmetrical inplane illumination (solid line) was applied without phase delay, the " $F$ " mode was weakened and the " $\mathrm{H}$ " mode was apparently enhanced ${ }^{42}$. As a comparison, when a phase delay of $\pi$ was applied in the symmetrical in-plane illumination, an opposite weakened/enhanced interference was observed for the two modes.

The weakened and enhanced phenomena are caused by the destructive and constructive plasmon resonances, which can be clearly demonstrated by the spatial distributions of electric-field amplitude for the gold nanodisk with a diameter of $160 \mathrm{~nm}$ (Fig. 2c and Supplementary Movie 1). The destructive and constructive interference can be further explained by the distributions of real and imaginary parts of electric-field component $E_{\mathrm{z}}$ for the "F" and " $\mathrm{H}$ " modes (Fig. 2d, e). More specifically, when $s$ polarized light comes only from the right side, the corresponding $\operatorname{Re}\left(E_{\mathrm{z}}\right)$ and $\operatorname{Im}\left(E_{\mathrm{z}}\right)$ distributions are identical ("ID") and opposite ("OP") for the "F" and " $\mathrm{H}$ " modes, respectively. Considering that the nanodisk is axisymmetric, when $s$-polarized light comes from the left side without phase delay, the corresponding $E_{\mathrm{z}}$ distribution will be mirrored with a negative sign along the central axis of the nanodisk $(x=0)$, i.e., $E_{\mathrm{z}}(-x)=-E_{\mathrm{z}}(x)$. As a result, 


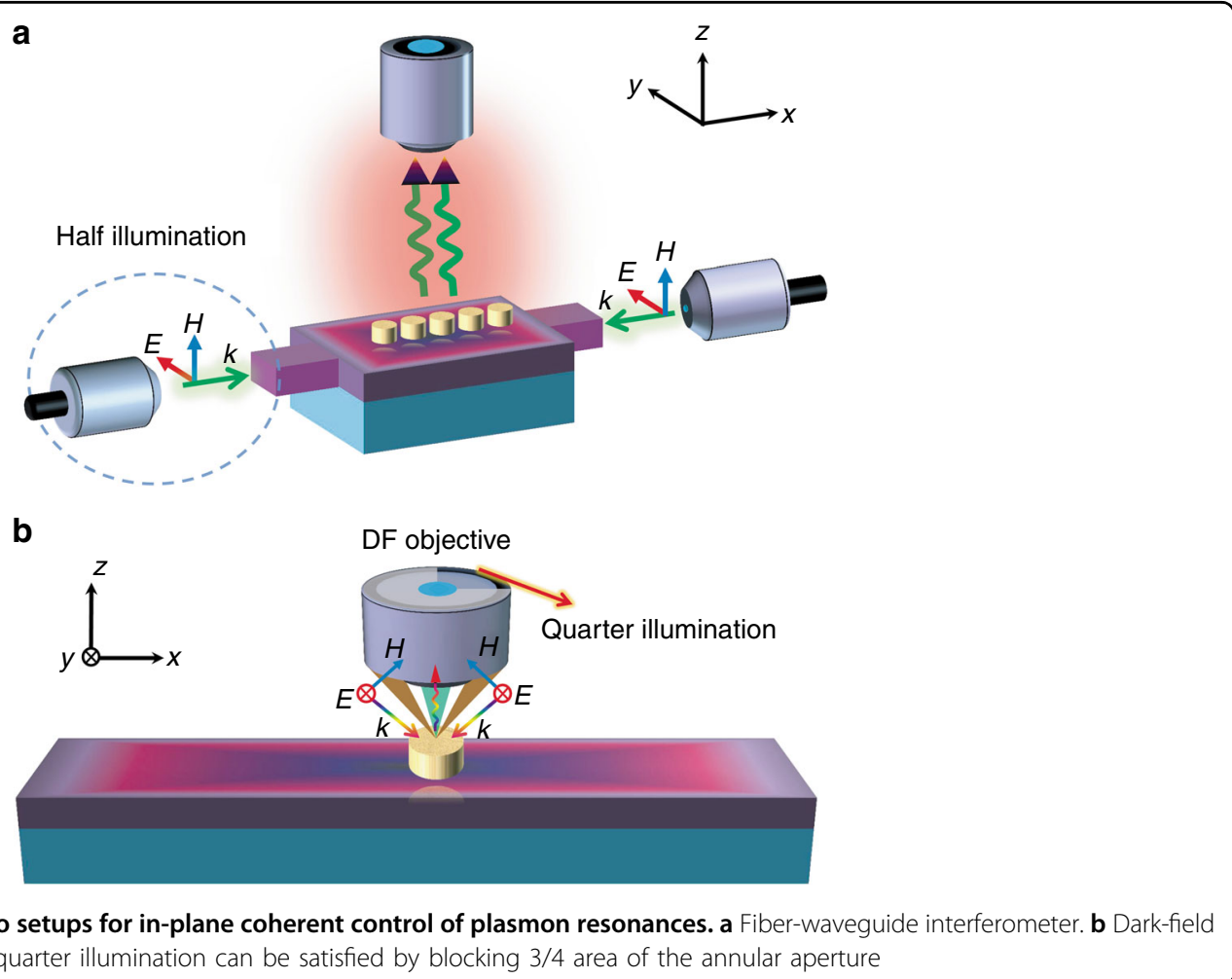

Fig. 1 Schematic diagrams of two setups for in-plane coherent control of plasmon resonances. a Fiber-waveguide interferometer. $\mathbf{b}$ Dark-field (DF) confocal microscope, where quarter illumination can be satisfied by blocking $3 / 4$ area of the annular aperture

the "F" mode with "ID" $\operatorname{Re}\left(E_{\mathrm{z}}\right)$ and $\operatorname{Im}\left(E_{\mathrm{z}}\right)$ distribution caused by right-side illumination will cancel with its negative mirror caused by left-side illumination and result in destructive interference, whereas the " $\mathrm{H}$ " mode with "OP" $\operatorname{Re}\left(E_{\mathrm{z}}\right)$ and $\operatorname{Im}\left(E_{\mathrm{z}}\right)$ distribution will superpose with its negative mirror and lead to constructive interference. As a comparison, when $s$-polarized light comes from the left side with a phase delay of $\pi$, the result of using a mirror operator is $E_{\mathrm{z}}(-x)=E_{\mathrm{z}}(x)$. As a result, under symmetrical illumination with a phase delay of $\pi$, the " $F$ " and " $\mathrm{H}$ " modes will experience constructive and destructive interference, respectively. For the case of $p$ polarized light, the phase delay-dependent mirror operators are consistent with the $s$-polarized light under symmetrical illumination. However, when $p$-polarized light comes only from the right side, the corresponding $\operatorname{Re}\left(E_{\mathrm{z}}\right)$ and $\operatorname{Im}\left(E_{\mathrm{z}}\right)$ distributions are opposite ("OP") and identical ("ID") for the "F" and " $H$ " modes, respectively. Therefore, the total phase delay-dependent destructive/constructive interference for the " $\mathrm{F}$ " and " $\mathrm{H}$ " modes are opposite to the case of $s$-polarization (Fig. S1 in Supplementary Information). The destructive/constructive interference of plasmon resonances was rechecked by the electromagnetic multipole theory, based on which the calculated absorption spectra of a $200 \mathrm{~nm}$ gold nanodisk monomer under symmetrical illumination matched well with the simulation results (Fig. S2 and Supplementary Note 1 in Supplementary Information). We have also studied the in- plane coherent control of plasmon resonances in gold nanodisk dimers, which show opposite phase delaydependent destructive/constructive interference for the "F" and " $\mathrm{H}$ " modes as compared with the monomers (Fig. S3 in Supplementary Information).

\section{Demonstration of electric-field distribution rule by s-SNOM}

Table 1 summarizes the distribution rule for all three electrical-field components under in-plane symmetric illumination, where the rule of destructive/constructive interference is always identical for $E_{\mathrm{y}}$ and $E_{\mathrm{z}}$, but opposite for $E_{\mathrm{x}}$. We used the polarization-sensitive scattering-type scanning near-field optical microscopy (s-SNOM) to verify the electric-field distribution rule. The s-SNOM measurement was conducted by a focused laser $(\lambda=$ $633 \mathrm{~nm}$ ) coming from one side with an incidence angle of $30^{\circ}$ with respect to the plane of the substrate, corresponding to an $s-s / s-p$ geometry scheme (Fig. 3a). For $s-s$ measurement, we recorded $E_{s-s}$ which is equal to $E_{\mathrm{y}}$. In $s-$ $p$ measurement, we detected the total electric field $E_{s-p}$, written by $\left(E_{\mathrm{x}}^{2}+E_{\mathrm{z}}^{2}\right)^{1 / 2}$, where the calculated amplitude of $E_{\mathrm{z}}$ is much larger than $E_{\mathrm{x}}$ and thus the total electric field $E_{s-p}$ is roughly equal to $E_{\mathrm{z}}$. As an example, $s-s$ and $s-$ $p$ excitation-collection measurements were carried out on a $200 \mathrm{~nm}$ gold nanodisk monomer and dimer with a gap size of $30 \mathrm{~nm}$. From Fig. 3b, we can find that the "H" mode at incidence angle $30^{\circ}$ is located near the excitation wavelength, and both the monomer and dimer show 


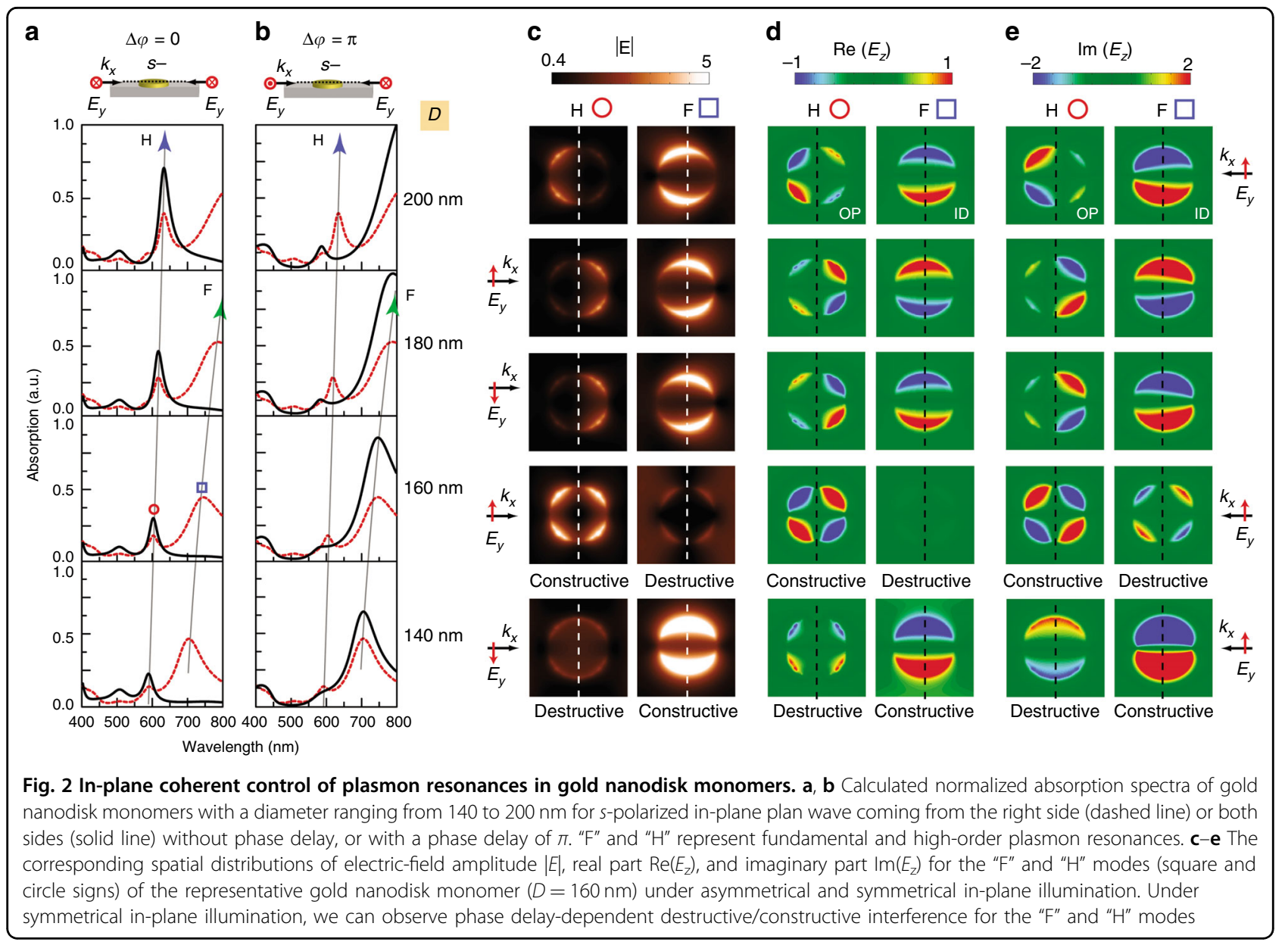

Table 1 Electric-field distribution rule for destructive and constructive plasmon resonances under symmetrical inplane illumination

\begin{tabular}{lllll}
\hline Phase delay & $\boldsymbol{s}$-/p-polarization & $\operatorname{Re} / \operatorname{Im}\left(E_{\mathbf{x}}\right)$ & $\operatorname{Re} / \operatorname{Im}\left(E_{\mathbf{y}}\right)$ & $\operatorname{Re} / \operatorname{Im}\left(E_{\mathbf{z}}\right)$ \\
\hline 0 & Destructive & OP & ID & ID \\
0 & Constructive & ID & OP & OP \\
$\pi$ & Destructive & ID & OP & OP \\
$\pi$ & Constructive & OP & ID & ID \\
\hline
\end{tabular}

constructive interference of " $\mathrm{H}$ " mode under symmetrical illumination without phase delay, which implies that both $\operatorname{Re}\left(E_{\mathrm{y}}\right)$ and $\operatorname{Re}\left(E_{\mathrm{z}}\right)$ should satisfy an "OP" distribution, according to Table 1 . Figure $3 \mathrm{~d}$ shows the measured and simulated amplitude, phase, and real part of $E_{\mathrm{y}}$ for the $s-s$ excitation-collection measurement, whereas the corresponding results of the $E_{\mathrm{z}}$ component for $s-p$ measurement are shown in Fig. 3e. Both measured and simulated results give strong evidence to demonstrate the "OP" distribution for both $\operatorname{Re}\left(E_{\mathrm{y}}\right)$ and $\operatorname{Re}\left(E_{\mathrm{z}}\right)$, where single and coupled quadrupole-like plasmon resonance can be observed for the monomer and dimer, respectively.

Plasmonic switching in gold nanodisk monomer and dimer

One promising application based on the in-plane coherent control of plasmon resonances is plasmonic switching. Here we employed the DF confocal microscopy (Fig. 1b) to experimentally demonstrate the plasmonic switching effect in a gold nanodisk monomer and dimer. During the measurement, a condenser lens with a high NA (0.9) was used to provide sufficient in-plane illumination and an opaque tape was used to construct the quarter illumination. Both $s$ - and $p$-polarization components of excitation were considered, and the collection was unpolarized. We experimentally observed the plasmonic switching effect in a $200 \mathrm{~nm}$ gold nanodisk monomer (Fig. 4a), i.e., constructive interference (area I) for the " $\mathrm{H}$ " plasmon resonance and destructive interference (area II) for the "F" plasmon resonance. Such a plasmonic switching phenomenon is clear and consistent for both $y$ - and $x$-polarization under full and quarter illumination, and is in good agreement with the simulated scattering and absorption spectra (Fig. 4b). The 


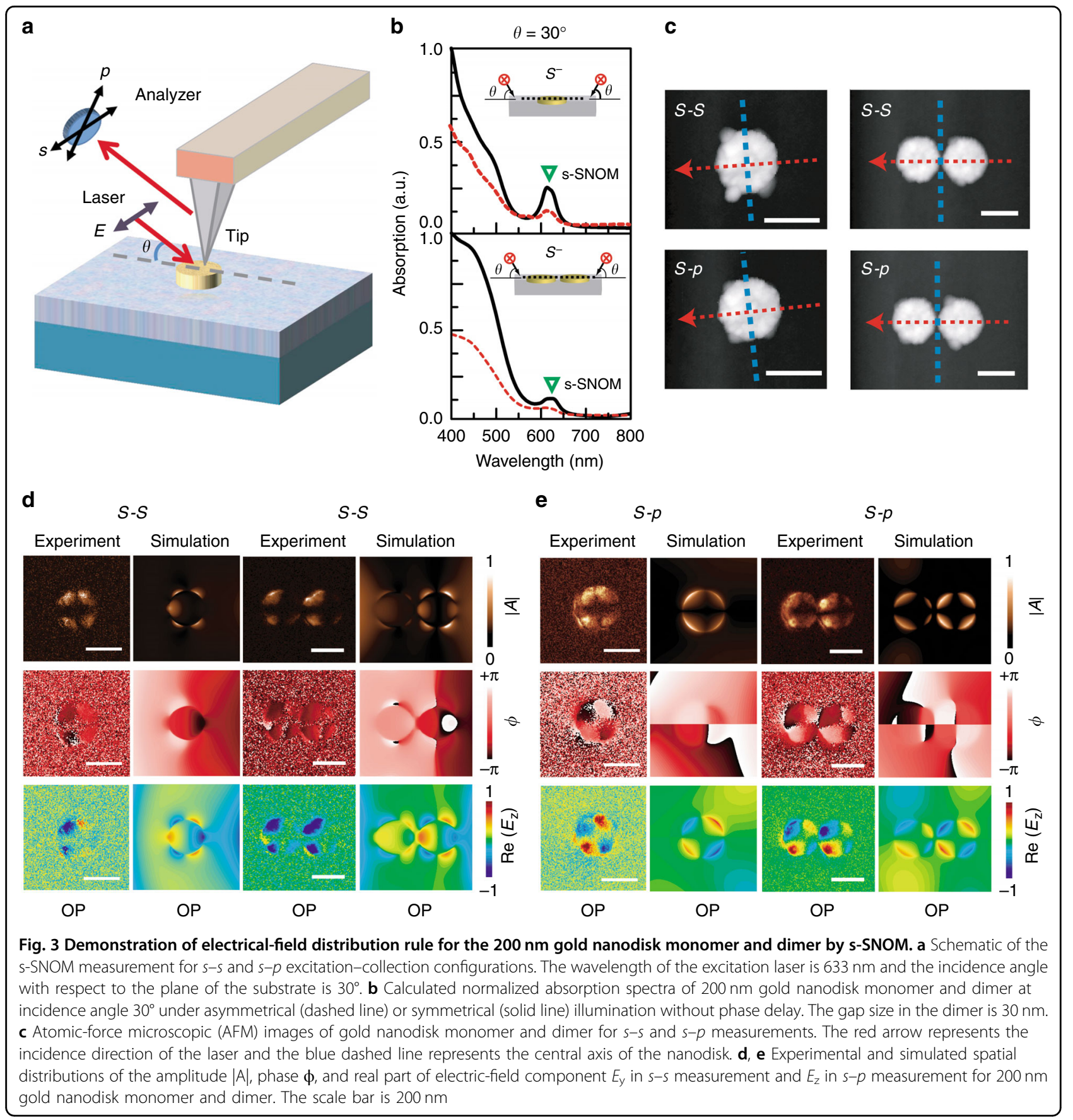

polarization diagrams for full and quarter illumination (Fig. 4e) indicate that the $y$ - and $x$-polarization of excitation are identical for the gold nanodisk monomer, resulting in an isotropic plasmonic switching effect. As a comparison, the plasmonic switching effect only occurs for the $x$-polarization of excitation in the gold nanodisk dimer, as shown in Fig. 4c, d. The anisotropic plasmonic switching effect in the gold nanodisk dimer is due to the superposition of anisotropic destructive and constructive plasmon resonances contributed by the in- plane wave vectors along the long and short axis, as shown in Fig. 4f.

\section{Plasmonic encoding in gold nanodisk chains}

Another possible application based on the in-plane coherent control of plasmon resonances is plasmonic encoding. Here we propose a plasmonic encoding strategy in a series of gold nanodisk chains consisting of different numbers of $140 \mathrm{~nm}$ nanodisks with a separation distance of $30 \mathrm{~nm}$. Figure $5 \mathrm{a}$ shows the absorption spectra of gold 


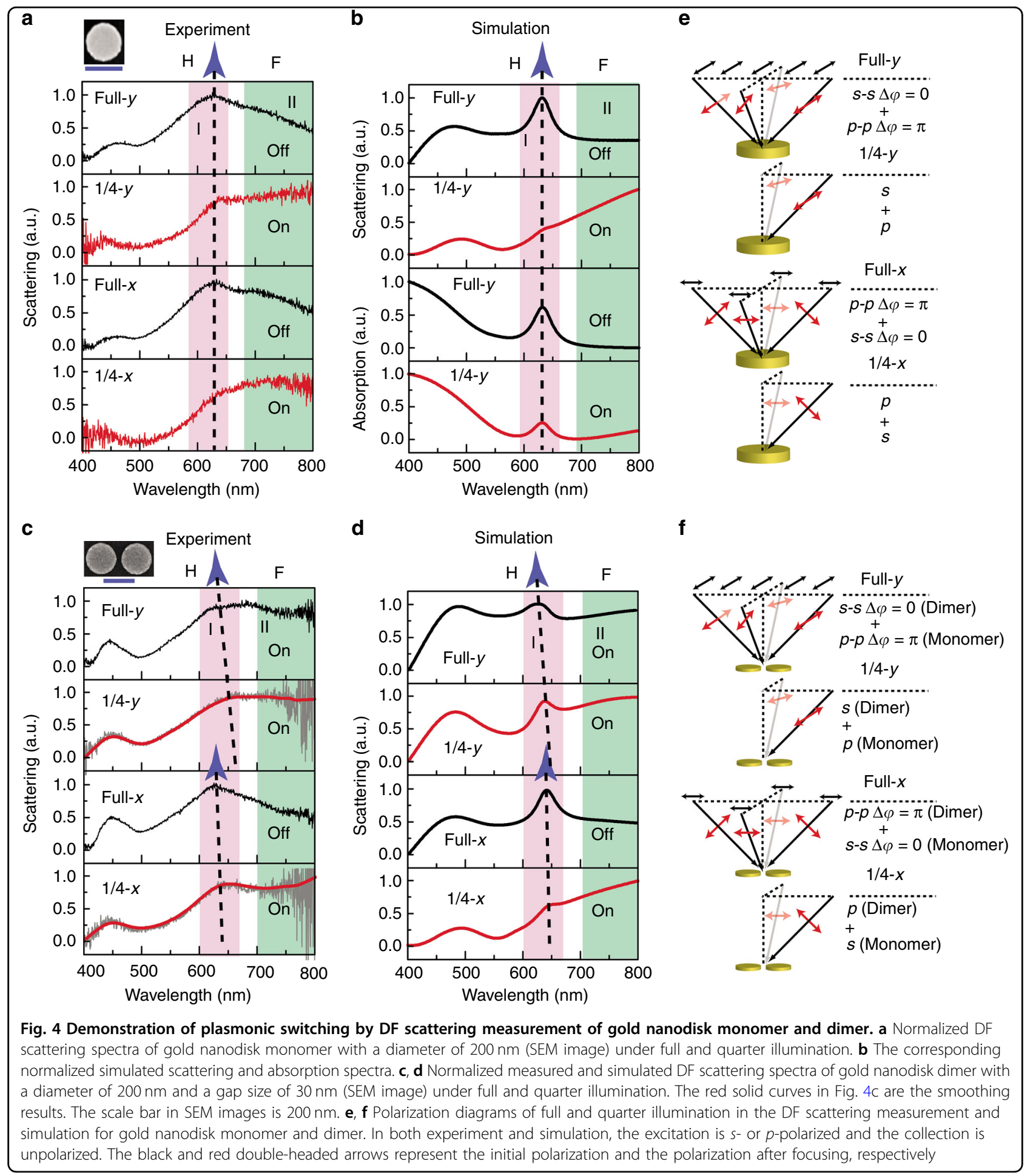

nanodisk chains under in-plane asymmetric (dashed line) or symmetrical (solid line) $s$-polarized illumination without phase delay. The " $\mathrm{F}$ " mode for gold nanodisk chains with number $N$ from 2 to 6 always shows constructive interference under the symmetrical illumination, whereas the " $\mathrm{H}$ " mode shows alternate constructive and destructive interference from nanodisk monomer to dimer, trimer, tetramer, and pentamer. Figure $5 \mathrm{~b}$ shows the spatial distributions of electric-field amplitude for the " $F$ " mode under symmetrical illumination, where the gold nanodisk chains show quite different propagating coupling behavior compared with that under asymmetric 


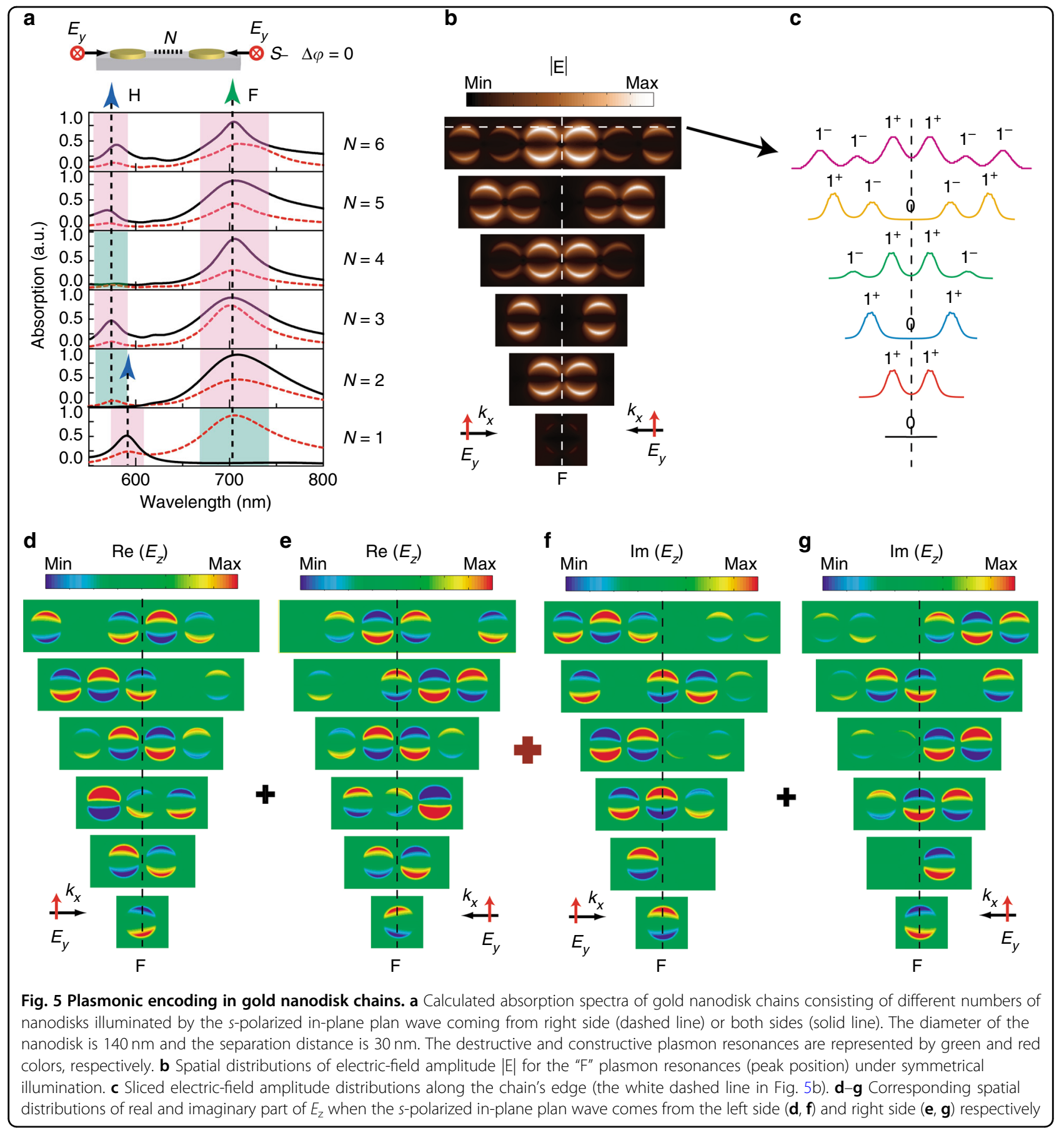

illumination. More specifically, under asymmetric illumination, the plasmonic coupling and propagation are along the long axis of the gold nanodisk chain and decrease in intensity (Fig. S4 and Supplementary Note 2 in Supplementary Information) ${ }^{42}$. Under symmetrical illumination, the plasmonic coupling and propagation are no longer continuous. The nanodisk in the center of chains with odd numbers of $N$ always shows completely destructive interference, whereas the two nanodisks in the center of chains with even numbers of $N$ always show constructive interference (see Supplementary Movie 2). The spatially related destructive and constructive interference inside the gold nanodisk chains can be used for plasmonic encoding, as shown in the sliced electric-field amplitude distributions in Fig. 5c, where signals $0,1^{-}$, and $1^{+}$can be generated based on the relative intensity along the nanodisk chain's edge. The plasmonic encoding can be well explained by the superposition of $\operatorname{Re}\left(E_{\mathrm{z}}\right)$ and $\operatorname{Im}$ $\left(E_{\mathrm{z}}\right)$ distributions under left- and right-side illumination, as shown in Fig. $5 \mathrm{~d}-\mathrm{g}$, where the destructive and 
constructive interference always occurs when the nanodisk satisfies "OP" and "ID" distributions, respectively. Besides the number of gold nanodisk chains, the input phase delay can also affect the plasmonic encoding. The signals 0 and $1^{+}$inside the gold nanodisk pentamer can be step-by-step shifted to the adjacent nanodisks by introducing an increasing phase delay from 0 to $3 \pi / 2$, with an interval of $\pi / 2$ (Fig. S5 and Supplementary Movie 3).

\section{Discussion}

In summary, we have demonstrated the idea of coherent control of plasmon resonances under symmetrical in-plane illumination. The coherent absorption for a single gold nanodisk shows a distinct mode-selection character due to destructive/constructive fundamental and higher-order plasmon resonances. We established and experimentally verified the distribution rule of electrical-field components for realizing destructive and constructive plasmon resonances in an axisymmetric plasmonic nanostructure. The constructive fundamental plasmon resonance for a gold nanodisk chain shows a distinct spatial-selection character due to phase-dependent coherent absorption along the chain. Compared with out-of-plane coherent control, inplane coherent control of plasmon resonances strongly relies on the configuration and symmetry of plasmonic nanostructures rather than the spatial position of metadevices placed in standing waves. This allows us more freedom in tailoring and engineering the multiple plasmon resonances in other axisymmetric plasmonic nanostructures, including nanosphere, nanorod, nano bowtie, and nanostructure polymer.

Besides the proof-of-principle demonstrations of plasmonic switching and encoding, more potential applications based on in-plane coherent control of plasmon resonances can be expected. Specifically, the plasmonic switching effect is ready to be extended to the study of selective surface-enhanced spectra, such as surfaceenhanced fluorescence ${ }^{43}$ and surface-enhanced Raman scattering ${ }^{44}$, during which the photoluminescence or Raman signal of multiple molecules can be selectively enhanced by controlling the on/off state of multiple plasmon resonances in one common nano-antenna ${ }^{45}$. A plasmonic encoding scheme can be extended to plasmonic imaging, nanolasing, and optical communication in nanocircuits. For instance, plasmonic nanostructure arrays doped with different fluorophore or gain materials can be used to realize selective plasmonic imaging ${ }^{46}$ or nanolasers ${ }^{12}$ through spatially selective coherent absorption in the array. By using combined plasmonic nanostructure chains with different encoding characteristics, logic units (such as XOR, NOT, and AND) and multichannel waveguides can be designed for all-optical information storage and processes ${ }^{35,36}$.

\section{Materials and methods}

Fabrication of gold nanodisks

We employed the electron-beam lithography (EBL) and a lift-off process to fabricate the gold nanodisk samples on $\mathrm{SiO}_{2} / \mathrm{Si}$ substrates ${ }^{47}$, where a $100 \mathrm{~nm}$-thick $\mathrm{SiO}_{2}$ layer was used for reducing plasmon damping. The gold film $(30 \mathrm{~nm}$ thick) and the underlying $\mathrm{Cr}$ adhesion layer $(1 \mathrm{~nm}$ thick) were deposited on the $\mathrm{SiO}_{2} / \mathrm{Si}$ substrate through the electron-beam evaporation. During the EBL process (Elionix ELS-7000), the accelerating voltage and beam current were set to $100 \mathrm{keV}$ and $100 \mathrm{pA}$, respectively. $N$ methylpyrrolidone solvent was used in the lift-off process and the solvent was heated up to $65^{\circ} \mathrm{C}$.

\section{s-SNOM imaging}

A polarization-sensitive s-SNOM was used to image the plasmon resonance modes in gold nanodisks based on a Neaspec commercial instrument, where a balanced technique was implemented for the optical-signal detec$\operatorname{tion}^{48}$. We used an $s-s / s-p$ geometry scheme and engaged a dielectric (Si) tip for measurements, during which $s$-polarized $\mathrm{CW}$ laser radiation $(\lambda=633 \mathrm{~nm})$ was used to illuminate the sample with an incidence angle of $30^{\circ}$ with respect to the plane of the substrate. The plasmonic mode images were recorded simultaneously with the disk's topography upon raster scanning of the sample. Here, the tip-scattered optical signal and tip-height position were recorded at each point of the scan. The amplitude and phase of the scattered signal were measured based on the pseudoheterodyne interferometric detection at the fourth harmonic of the tip-tapping frequency. An analyzer placed in front of the detector was used to select the $p$ - or $s$-polarized component of the scattered light.

\section{DF scattering measurement}

A confocal Raman microscopy system (WITec CRM200) was used to measure the DF scattering spectra of gold nanodisk samples. A high-power halogen lamp (Philips, $100 \mathrm{~W}$ ) was used as the light source. The scattering light was collected by a $\times 100 \mathrm{DF}$ objective lens (Zeiss Epiplan, NA $=0.9$ ) and the final spectra was generated by a TE-cooled charge-coupled device (Andor DV 401-BV-351) with a 150 line/mm grating in front of it. In the quarter-illumination measurement, the input of a condensed DF lens was blocked in three-fourth area by an opaque tape. In all measurements of scattering spectra, the integration time was $20 \mathrm{~s}$.

\section{Numerical simulations}

We employed a commercial software package (Lumerical FDTD Solutions) to simulate the electrical-field distributions, absorption, and scattering spectra of the gold nanodisks. For $x, y$, and $z$ directions, the boundary 
conditions were set to perfectly matched layer and the finest mesh size in the structure was set to $1 \mathrm{~nm}$. In Figs. 2 and 5 , the simulation was done with a pure $\mathrm{SiO}_{2} / \mathrm{Si}$ substrate, whereas the simulation also contained the $\mathrm{Cr}$ adhesion in Figs. 3 and 4. The complex electromagnetic parameters were from Johnson and Christy for $\mathrm{Au}$ and $\mathrm{Cr}$, and from Palik for $\mathrm{SiO}_{2}$ and $\mathrm{Si}^{49}$.

\section{Acknowledgements}

L.Y.J., T.T.Y., and A.M.D. acknowledge the funding support from Singapore Ministry of Education Academic Research Fund Tier 2 (grant number MOE2012-T2-2-124) and Tier 3 (grant number MOE2011-T3-1-005). L.Y.J. also acknowledges the funding support from the National Natural Science Foundation of China (grant numbers 61675096 and 61205042) and the Natural Science Foundation of Jiangsu Province in China (grant number BK20141393). Z.G.D. and J.K.W.Y. acknowledge the funding support from Agency for Science, Technology and Research (A*STAR) SERC Pharos project (grant number 1527300025), and A*STAR-JCO under project number 1437C00135. We greatly thanks Professor N. I. Zheludev, Professor Y. D. Chong, and Professor R. Singh for their good suggestions on this work. We also thank Dr. Chen Qian and Dr. Bo Qiang for their help in the experiments and simulation.

\section{Author details \\ 'Department of Physics, School of Science, Nanjing University of Science and Technology, Nanjing 210094, China. ${ }^{2}$ Centre for Disruptive Photonic Technologies, The Photonics Institute, School of Physical and Mathematical Sciences, Nanyang Technological University, 21 Nanyang Link, Singapore 637371, Singapore. ${ }^{3}$ Institute of Materials Research and Engineering, A*STAR (Agency for Science, Technology and Research), \#08-03 Innovis, Singapore 138634, Singapore. ${ }^{4}$ School of Optical and Electronic Information, Huazhong University of Science and Technology, Wuhan 430074, China. ${ }^{5}$ Singapore University of Technology and Design, 8 Somapah Road, Singapore 487372, Singapore}

\section{Author contributions}

L.Y.J. proposed the idea and conducted the theoretical analysis and simulation. T.T.Y. conducted the dark-field measurement. A.M.D. conducted the s-SNOM measurement. Z.G.D. fabricated the gold nanodisk samples. Y.T.C. and W.J.C. contributed to the theoretical calculation based on electromagnetic multipole theory. L.Y.J. drafted the manuscript. All authors contributed to the interpretation of results. Z.X.S. and J.K.W.Y. supervised the work.

\section{Conflict of interest}

The authors declare that they have no conflict of interest.

Supplementary information is available for this paper at https://doi.org/ 10.1038/s41377-019-0134-1.

Received: 30 October 2018 Revised: 29 December 2018 Accepted: 17 January 2019

Published online: 06 February 2019

\section{References}

1. Maier, S. A. Plasmonics: Fundamentals And Applications (Springer, 2007).

2. GarciaVidal, F. J. \& Pendry, J. B. Collective theory for surface enhanced Raman scattering. Phys. Rev. Lett. 77, 1163-1166 (1996).

3. Lin, K. Q. et al. Plasmonic photoluminescence for recovering native chemical information from surface-enhanced Raman scattering. Nat. Commun. 8, 14891 (2017).

4. Maier, S. A. et al. Local detection of electromagnetic energy transport below the diffraction limit in metal nanoparticle plasmon waveguides. Nat. Mater. $\mathbf{2}$, 229-232 (2003).

5. Sonnichsen, C., Reinhard, B. M., Liphardt, J. \& Alivisatos, A. P. A molecular ruler based on plasmon coupling of single gold and silver nanoparticles. Nat. Biotechnol. 23, 741-745 (2005).
6. Anker, J. N. et al. Biosensing with plasmonic nanosensors. Nat. Mater. 7, 442-453 (2008).

7. $\mathrm{Wu}, \mathrm{X}$. et al. High-photoluminescence-yield gold nanocubes: for cell imaging and photothermal therapy. ACS Nano 4, 113-120 (2010).

8. Tanaka, K, Plum, E., Ou, J. Y., Uchino, T. \& Zheludev, N. I. Multifold enhancement of quantum dot luminescence in plasmonic metamaterials. Phys. Rev. Lett. 105, 227403 (2010).

9. Wang, Z. et al. Giant photoluminescence enhancement in tungstendiselenide-gold plasmonic hybrid structures. Nat. Commun. 7, 11283 (2016).

10. Noginov, M. A. et al. Demonstration of a spaser-based nanolaser. Nature $\mathbf{4 6 0}$ 1110-U1168 (2009).

11. Oulton, R. F. et al. Plasmon lasers at deep subwavelength scale. Nature $\mathbf{4 6 1}$ 629-632 (2009).

12. Yang, A. K. et al. Real-time tunable lasing from plasmonic nanocavity arrays. Nat. Commun. 6, 6939 (2015).

13. Kumar, K. et al. Printing colour at the optical diffraction limit. Nat. Nanotechnol. 7, 557-561 (2012)

14. Guay, J. M. et al. Laser-induced plasmonic colours on metals. Nat. Commun. 8, 16095 (2017)

15. Tan, S. F. et al. Quantum plasmon resonances controlled by molecular tunnel junctions. Science 343, 1496-1499 (2014)

16. Zheng, G. X. et al. Metasurface holograms reaching $80 \%$ efficiency. Nat. Nanotechnol. 10, 308-312 (2015)

17. Huang, L. L. et al. Three-dimensional optical holography using a plasmonic metasurface. Nat. Commun. 4, 2808 (2013).

18. Wang, S. M. et al. Broadband achromatic optical metasurface devices. Nat. Commun. 8, 187 (2017).

19. Mock, J. J., Barbic, M., Smith, D. R., Schultz, D. A. \& Schultz, S. Shape effects in plasmon resonance of individual colloidal silver nanoparticles. J. Chem. Phys. 116, 6755-6759 (2002).

20. Kelly, K. L., Coronado, E., Zhao, L. L. \& Schatz, G. C. The optical properties of metal nanoparticles: the influence of size, shape, and dielectric environment. J. Phys. Chem. B 107, 668-677 (2003).

21. Rechberger, $W$. et al. Optical properties of two interacting gold nanoparticles Opt. Commun. 220, 137-141 (2003).

22. Halas, N. J., Lal, S., Chang, W. S., Link, S. \& Nordlander, P. Plasmons in strongly coupled metallic nanostructures. Chem. Rev. 111, 3913-3961 (2011).

23. Gans, R. The state of ultramicroscopic silver particles. Ann. Phys. Berl. 47, 270-U214 (1915).

24. Nordlander, P., Oubre, C., Prodan, E., Li, K. \& Stockman, M. I. Plasmon hybridizaton in nanoparticle dimers. Nano Lett. 4, 899-903 (2004).

25. Prodan, E., Radloff, C., Halas, N. J. \& Nordlander, P. A hybridization model for the plasmon response of complex nanostructures. Science 302, 419-422 (2003).

26. Jain, P. K. \& El-Sayed, M. A. Plasmonic coupling in noble metal nanostructures. Chem. Phys. Lett. 487, 153-164 (2010).

27. Luk'yanchuk, B. et al. The Fano resonance in plasmonic nanostructures and metamaterials. Nat. Mater. 9, 707-715 (2010).

28. Liu, N. et al. Plasmonic analogue of electromagnetically induced transparency at the Drude damping limit. Nat. Mater. 8, 758-762 (2009).

29. Link, S. \& Ei-Sayed, M. A. Optical properties and ultrafast dynamics of metallic nanocrystals. Annu. Rev. Phys. Chem. 54, 331-366 (2003).

30. Kim, S. et al. High-harmonic generation by resonant plasmon field enhancement. Nature 453, 757-760 (2008).

31. Tame, M. S. et al. Quantum plasmonics. Nat. Phys. 9, 329-340 (2013).

32. Wan, W. J. et al. Time-reversed lasing and interferometric control of absorption. Science 331, 889-892 (2011).

33. Roger, $T$. et al. Coherent perfect absorption in deeply subwavelength films in the single-photon regime. Nat. Commun. 6, 7031 (2015).

34. Zhang, J. F., MacDonald, K. F. \& Zheludev, N. I. Controlling light-with-light without nonlinearity. Light Sci. Appl. 1, e18 (2012).

35. Fang, X., MacDonald, K. F. \& Zheludev, N. I. Controlling light with light using coherent metadevices: all-optical transistor, summator and invertor. Light SCi. Appl. 4, e292 (2015)

36. Xomalis, A. et al. Fibre-optic metadevice for all-optical signal modulation based on coherent absorption. Nat. Commun. 9, 182 (2018).

37. Fan, J. A. et al. Self-assembled plasmonic nanoparticle clusters. Science $\mathbf{3 2 8}$ $1135-1138$ (2010) 
38. Knight, M. W., Fan, J., Capasso, F. \& Halas, N. J. Influence of excitation and collection geometry on the dark field spectra of individual plasmonic nanostructures. Opt. Express 18, 2579-2587 (2010).

39. Jiang, L. Y. et al. Accurate modeling of dark-field scattering spectra of plasmonic nano structures. ACS Nano 9, 10039-10046 (2015).

40. Fan, J. A. et al. Near-normal incidence dark-field microscopy: applications to nanoplasmonic spectroscopy. Nano Lett. 12, 2817-2821 (2012).

41. Kottmann, J. P. \& Martin, O. J. F. Retardation-induced plasmon resonances in coupled nanoparticles. Opt. Lett. 26, 1096-1098 (2001).

42. Maier, S. A. et al. Plasmonics - a route to nanoscale optical devices. Adv. Mater. 13, 1501-1505 (2001).

43. Jiang, L. Y. et al. Probing vertical and horizontal plasmonic resonant states in the photoluminescence of gold nanodisks. ACS Photonics 2, 1217-1223 (2015).
44. Zhang, R. et al. Chemical mapping of a single molecule by plasmon-enhanced Raman scattering. Nature 498, 82-86 (2013).

45. Coenen, T., Arango, F. B., Koenderink, A. F. \& Polman, A. Directional emission from a single plasmonic scatterer. Nat. Commun. 5, 3250 (2014).

46. Dregely, D. et al. Imaging and steering an optical wireless nanoantenna link. Nat. Commun. 5, 4354 (2014).

47. Dong, Z. G., Bosman, M., Zhu, D., Goh, X. M. \& Yang, J. K. W. Fabrication of suspended metal-dielectric-metal plasmonic nanostructures. Nanotechnology 25, 135303 (2014)

48. Dubrovkin, A. M. et al. Visible range plasmonic modes on topological insulator nanostructures. Adv. Opt. Mater. 5, 1600768 (2017).

49. Johnson, P. B. \& Christy, R. W. Optical constants of the noble metals. Phys. Rev. B 6, 4370-4379 (1972). 